PROCEEDINGS OF THE

AMERICAN MATHEMATICAL SOCIETY

Volume 132, Number 2, Pages 535-541

S 0002-9939(03)07054-0

Article electronically published on June 11, 2003

\title{
MAXIMAL ELEMENTS IN NONCOMPACT SPACES WITH APPLICATION TO EQUILIBRIA
}

\author{
SHIOW-YU CHANG
}

(Communicated by Joseph A. Ball)

\begin{abstract}
A new maximal theorem for $L_{S}$-majorized correspondences in noncompact spaces is presented and applied to obtain an equilibrium existence theorem for noncompact abstract economies. The corresponding results of Borglin and Keiding (1976), Yannelis and Prabhakar (1983), Ding and Tan (1993), Yuan and Tarafdar (1996), and Ding and Yuan (1998) are generalized by our results.
\end{abstract}

\section{INTRODUCTION}

The existence of a Cournot-Nash equilibrium for a normal form game was proved by Nash (1951). The notion of an abstract economy (social system) was introduced by Debreu (1952) which contains the normal game form as a special case and proved the existence of an equilibrium. The equilibrium result of Debreu not only provides as a corollary the Nash existence theorem, but it was also the instrument used by Arrow-Debreu (1954) to prove the existence of a Walrasian equilibrium. The Debreu theorem was generalized by Shafer-Sonnenschein (S-S) and Borglin-Keiding (B-K) to allow for preferences which need not be representable by utility functions. All the above results are for finite dimensions and do not allow for an infinite number of agents. Most importantly however, as was remarked by Yannelis-Prabhakar (Y-P), the proofs of S-S and B-K fail in the presence of an infinite number of commodities or an infinite number of agents, and new arguments are required. To this end not only were new mathematical results proved in Y-P, but also a new class of correspondences was introduced called L-class.

In this paper, we extend the L-class further and provide new results on maximal elements and equilibrium by dispensing with the assumption of compact strategy sets which generalizes the results in Y-P. We mention that such attempts were made by Yannelis (1985) for maximal elements over noncompact subsets of linear topological spaces.

The corresponding results of Fan (1962), Borglin and Keiding (1976), Yannelis and Prabhakar (1983), Yannelis (1985), Chang (1989) (1990), Kim (1992), Ding and Tan (1993), Yuan and Tarafdar (1996), and Ding and Yuan (1998) are generalized by our results.

Received by the editors January 30, 2002 and, in revised form, September 30, 2002. 2000 Mathematics Subject Classification. Primary 91A13; Secondary 52A07, 91B50.

Key words and phrases. Maximal element, abstract economy, $L_{S}$-majorized correspondences. 


\section{NotATION AND DEFINITIONS}

(1) $2^{A} \quad$ denotes the set of all subsets of $A$.

(2) $\operatorname{co} A$ denotes the convex hull of the set $A$.

(3) $A / B$ denotes the difference of sets $A$ and $B$.

(4) $\operatorname{cl} A$, int $A$ denote the closure and interior of the set $A$.

(5) $\phi: X \rightarrow 2^{Y}$ is a correspondence, that is, $\phi(x)$ is a subset of $Y$ for each $x \in X$.

(6) Let $X=\prod_{i \in I} X_{i}$ and $\pi_{j}: X \rightarrow X_{j}$ be called the projection of $X$ onto $X_{j}$, if $\pi_{j}(x)=x_{j}$ for each $x=\left(x_{i}\right)_{i \in I} \in X$.

Let $I$ be a (possibly uncountable) set. For each agent $i \in I$, let its choice set or strategy set $X_{i}$ be a nonempty set in a topological vector space. Let $X=\prod_{j \in I} X_{j}$. Following Gale and Mas-Colell [9], $\Gamma=\left(X_{i}, P_{i}\right)_{i \in I}$ is a qualitative game if for each player $i \in I, X_{i}$ is the strategy set of player $i$, and $P_{i}: X=\prod_{j \in I} X_{j} \rightarrow 2^{X_{i}}$ is a preference correspondence of player $i$ which is irreflexive [i.e. $\pi_{i}(x) \notin P_{i}(x)$ for each $x \in X]$; also, a point $\tilde{x} \in X$ is said to be an equilibrium point of the game $\Gamma=\left(X_{i}, P_{i}\right)_{i \in I}$ if $P_{i}(\tilde{x})=\emptyset$ for each $i \in I$. A generalized model is as follows:

Definition 1. Let $I$ denote the set of agents. For each $i \in I$, let $X_{i}$ be a nonempty set and $X=\prod_{j \in I} X_{j}$. Following Ding et al. [6], an abstract economy or (generalized game) $G=\left(X_{i}, A_{i}, B_{i}, P_{i}\right)_{i \in I}$ is defined as a family of quadruples $\left(X_{i}, A_{i}, B_{i}, P_{i}\right)$, where $A_{i}, B_{i}: X \rightarrow 2^{X_{i}}$ are feasible correspondences of agent $i$ and $P_{i}: X \rightarrow 2^{X_{i}}$ are preference correspondences of agent $i$. An equilibrium point for $G$ is an $\tilde{x} \in X$ where $\tilde{x}=\left(\tilde{x}_{i}\right)$ satisfies

(1) $\tilde{x}_{i} \in B_{i}(\tilde{x})$,

(2) $P_{i}(\tilde{x}) \cap A_{i}(\tilde{x})=\emptyset$,

for each $i \in I$. When $A_{i}=B_{i}$ for each $i \in I$ our definition coincides with the standard definition, e.g. in Borglin and Keiding [2] or in Yannelis and Prabhakar [13].

Definition 2. Let $X$ be a topological space, $Y$ a nonempty subset of a vector space $E, \theta: X \rightarrow E$ a map and $\phi: X \rightarrow 2^{Y}$ a correspondence. The following notions were introduced in [2], [6]-[10, [12]-15]:

(1) A set $G \subset X$ is said to be compactly open in $X$ if for each compact set $K$ in $X$ the set $G \cap K$ is open in $K$.

(2) $\phi$ is said to have compactly open lower sections in $X$ if for each $y \in Y$ the set $\phi^{-1}(y)=\{x \in X \mid y \in \phi(x)\}$ is compactly open in $X$.

(3) $\phi$ is said to have locally compactly open lower section at $x$ if there is an open set $W_{x}$ containing $x$ such that for each $y \in Y$ the set $\phi^{-1}(y) \cap W_{x}$ is compactly open in $X$.

(4) $\phi$ is said to be of class $L_{\theta, S}$ if for every $x \in X, \theta(x) \notin \operatorname{co} \phi(x)$, and $\phi$ has compactly open lower sections in $X$.

(5) A correspondence $\phi_{x}: X \rightarrow 2^{Y}$ is said to be an $L_{\theta, S}$-majorant of $\phi$ at $x \in X$ if there exists an open neighborhood $N_{x}$ of in $X$ such that (a) for each $z \in N_{x}, \phi(z) \subset \phi_{x}(z)$ and $\theta(z) \notin \operatorname{co}_{x}(z)$, (b) for each $y \in Y, \phi_{x}^{-1}(y)$ is compactly open in $X$;

(6) Suppose $X^{\prime} \subset X$. $\phi$ is $L_{\theta, S}$-majorized in $X^{\prime}$ if for each $x \in X^{\prime}$ with $\phi(x) \neq \emptyset$ there exists an $L_{\theta, S}$-majorant of $\phi$ at $x$ in $X$. 
In this paper, we deal mainly with the case (I) $X=Y$ which is a nonempty convex subset of the topological vector space $E$ and $\theta=I_{X}$, the identity map on $X$, or the case (II) $X=\prod_{i \in I} X_{i}$ and $\theta=\pi_{j}: X \rightarrow X_{j}$ is the projection of $X$ onto $X_{j}$ and $Y=X_{j}$ is a nonempty convex subset of a topological vector space. In both cases (I) and (II), we write $L_{S}$ in place of $L_{\theta, S}$.

\section{THE EXISTENCE OF MAXIMAL ELEMENTS IN NONCOMPACT SPACES}

Let $X$ be a nonempty subset of a topological space and $U$ be a preference correspondence on $X$, that is, for each $x \in X$ associates a set $U(x) \subset X$, which may be interpreted as the set of those objects in $X$ that are "better", "larger" or "after" $x$. An element $x \in X$ is $U$-maximal if $U(x)=\emptyset$.

Theorem 5.1 of Yannelis and Prabhakar [14] is needed in order to prove the existence of maximal elements in noncompact spaces. This is given below in Theorem 1.

Theorem 1. Let $X$ be a compact convex set in a Hausdorff topological vector space and let $U: X \rightarrow 2^{X}$ satisfy the following conditions:

(1) $x \notin \operatorname{coU}(x)$ for all $x \in X$.

(2) $U^{-1}(x)=\{y \in X \mid x \in U(y)\}$ is open in $X$ for each $x \in X$.

Then $\{x \in X \mid U(x)=\emptyset\}$ is nonempty and compact.

The following result is a new theorem for the existence of maximal elements for $L_{S}$-majorized correspondences in noncompact spaces.

Theorem 2. Let $X$ be a convex subset of a Hausdorff topological vector space $E$ and let $U: X \rightarrow 2^{X}$ be everywhere $L_{S}$-majorized. Suppose there exists a compact set $L$ of $X$ such that for each finite subset $S$ of $X$, there is a compact convex set $K$ containing $S$ and satisfying

$$
K / \bigcup_{x \in K} U^{-1}(x) \subset L
$$

Then $\{x \in X \mid U(x)=\emptyset\}$ is a nonempty subset of $L$.

Proof. Suppose that $U(x) \neq \emptyset$ for each $x \in L$. Then by the hypothesis, $U(x) \neq \emptyset$ for each $x \in X$. For each $x \in X$, choose an open neighborhood $N_{x}$ of $x$ and $\phi_{x}: X \rightarrow 2^{X}$ such that (1) for each $x^{\prime} \in N_{x}, U\left(x^{\prime}\right) \subset \phi_{x}\left(x^{\prime}\right), x^{\prime} \notin \operatorname{co} \phi_{x}\left(x^{\prime}\right)$ and (2) for each $y \in X, \phi_{x}^{-1}(y)$ is compactly open. Let $\mathcal{U}=\left\{N_{x} \mid x \in X\right\}$. For each $x \in L$, choose $\tilde{N}_{x}$ to be a closed neighborhood of $x$ such that $\tilde{N}_{x} \subset N_{x}$. Since $L$ is compact, there exists a finite cover $\left\{\tilde{N}_{x_{1}}, \cdots, \tilde{N}_{x_{n}}\right\}$ such that $L$ is contained in the interior of $\bigcup_{i=1}^{n} \tilde{N}_{x_{i}}$. Let $F_{1}=\bigcup_{i=1}^{n} \tilde{N}_{x_{i}}$. Define $\psi_{i}: X \rightarrow 2^{X}, i=1, \cdots, n$, by

$$
\psi_{i}(x)= \begin{cases}\phi_{x_{i}}(x), & \text { if } x \in \tilde{N}_{x_{i}} \\ X, & \text { otherwise }\end{cases}
$$

Define $P_{1}$ on $X$ by $P_{1}(x)=\bigcap_{i=1}^{n} \psi_{x_{i}}(x)$. Then (1) for each $x^{\prime} \in F_{1}, U\left(x^{\prime}\right) \subset$ $P_{1}\left(x^{\prime}\right), x^{\prime} \notin \operatorname{co} P_{1}\left(x^{\prime}\right)$ and $(2)$ for each $y \in X, P_{1}^{-1}(y)$ is compactly open in $X$. Choose $y_{1}, \cdots, y_{m} \in X$ such that

$$
L \subset \bigcup_{i=1}^{m} P_{1}^{-1}\left(y_{i}\right)
$$


According to the hypothesis, there is a compact convex subset $K$ containing $y_{1}, \cdots, y_{m}$ such that

$$
K / \bigcup_{x \in K} U^{-1}(x) \subset L
$$

Let $X^{\prime}=c o(L \cup K)$ and $O$ be an open set containing $L$ such that its closure $\bar{O} \subset F_{1}$. Then $X^{\prime}$ is paracompact. For each $x \in X^{\prime} / O$, there exists a closed neighborhood $\tilde{N}_{x}$ of $x$ such that $\tilde{N}_{x} \subset N_{x}$ where $N_{x} \in \mathcal{U}$ and $\tilde{N}_{x} \bigcap L=\emptyset$. Since $X^{\prime} / O$ is paracompact, we can assume that there is a subset $D \subset X^{\prime} / O$ such that $\left\{\tilde{N}_{x} \mid x \in D\right\}$ is a locally finite closed cover of $X^{\prime} / O$. Let $I(y)=\left\{x \in D \mid y \in \tilde{N}_{x}\right\}$. Define $P_{2}:\left[X^{\prime} / O\right] \rightarrow X$ by $P_{2}(y)=\bigcap_{x \in I(y)} \phi_{x}(y)$. Then (1) for each $x^{\prime} \in$ $\left[X^{\prime} / O\right], U\left(x^{\prime}\right) \subset P_{2}\left(x^{\prime}\right), x^{\prime} \notin c o P_{2}\left(x^{\prime}\right)$ and (2) for each $y \in X, P_{2}^{-1}(y)$ is compactly open in $\left[X^{\prime} / O\right]$. Define $P: X^{\prime} \rightarrow 2^{X}$ by

$$
P(x)= \begin{cases}P_{1}(x) \cap P_{2}(x), & \text { if } x \in F_{1} \cap\left[X^{\prime} / O\right] ; \\ P_{1}(x), & \text { if } x \in O \cap X^{\prime} ; \\ P_{2}(x), & \text { if } x \in\left[X^{\prime} / F_{1}\right] .\end{cases}
$$

Then (1) for each $x^{\prime} \in X^{\prime}, U\left(x^{\prime}\right) \subset P\left(x^{\prime}\right), x^{\prime} \notin \operatorname{co} P\left(x^{\prime}\right)$ and (2) for each $y \in X$, $P^{-1}(y)$ is compactly open in $X^{\prime}$. Furthermore, for each $x \in L, P(x)=P_{1}(x)$ and hence by $(*)$ for each $x \in L$,

$$
(* * *) \quad P(x) \cap K \neq \emptyset \text {. }
$$

Then let $P_{K}: K \rightarrow 2^{K}$ be defined by $P_{K}(x)=P(x) \cap K$ for each $x \in K$. Then using Theorem 1, there exists $x_{0} \in K$ such that $P_{K}\left(x_{0}\right)=\emptyset$. Since $K \cap\left(\bigcup_{x \in K} U^{-1}(x)\right) \subset$ $\bigcup_{x \in K} P_{K}^{-1}(x)$, it follows that

$$
K / \bigcup_{x \in K} P_{K}^{-1}(x) \subset K / \bigcup_{x \in K} U^{-1}(x)
$$

and hence by $(* *), x_{0} \in L$. This contradicts $(* * *)$, therefore there is $x \in L$ such that $U(x)=\emptyset$.

Corollary 1. Let $X$ be a convex subset of a Hausdorff topological vector space $E$ and let $U: X \rightarrow 2^{X}$ be everywhere $L_{S}$-majorized. Suppose there exists a compact set $L$ and a compact convex set $K_{0}$ of $X$ such that for each $x \in X$

$$
c o\left(K_{0} \cup\{x\}\right) / \bigcup_{x^{\prime} \in \operatorname{co}\left(K_{0} \cup\{x\}\right)} U^{-1}\left(x^{\prime}\right) \subset L .
$$

Then $\{x \in X \mid U(x)=\emptyset\}$ is a nonempty subset of $L$.

Remark 1. Corollary 1 is an extension of Corollary 5.1 of Yannelis and Prabhakar 14 from compact spaces to noncompact spaces. It also extends Theorem 1 of Kim [10] from intersecting one fixed point to intersecting some compact convex set, and it extends Theorem 1 of Ding and Tan 7] and Theorem 2.3 of Ding and Yuan [8] from paracompact spaces to noncompact spaces.

\section{Equilibria of the generalized game}

For an application of Theorem 2, we prove the following existence theorem of equilibrium for an abstract economy with an infinite number of agents in noncompact topological vector spaces. 
Theorem 3. Let $\left(X_{i}, A_{i}, B_{i}, P_{i}\right)_{i \in I}$ be an abstract economy and $D$ be a compact subset of $X=\prod_{i \in I} X_{i}$. Suppose the following conditions are satisfied:

(1) $X_{i}$ is a nonempty convex subset of a Hausdorff topological vector space for each $i \in I$;

(2) for each $x \in X, A_{i}(x)$ is nonempty and $\operatorname{co} A_{i}(x) \subset B_{i}(x)$ for each $i \in I$;

(3) $\triangle_{i}:=\left\{x \in X \mid x_{i} \in B_{i}(x)\right\}$ is closed in $X$ for each $i \in I$;

(4) $A_{i}: X \rightarrow 2^{X_{i}}$ has locally compactly open lower sections in $\triangle_{i}$ and is $L_{S^{-}}$ majorized in $X / \triangle_{i}$ for each $i \in I$;

(5) $A_{i} \cap P_{i}: X \rightarrow 2^{X_{i}}$ is $L_{S}$-majorized in $\triangle_{i}$ for each $i \in I$;

(6) $\bigcap_{i \in I}\left\{x \in \triangle_{i} \mid\left(P_{i} \cap A_{i}\right)(x)=\emptyset\right\}=\bigcap_{i \in I} \operatorname{cl}\left\{x \in \triangle_{i} \mid\left(P_{i} \cap A_{i}\right)(x)=\emptyset\right\}$. Moreover, for each finite set $S \subset X$, there exists a compact convex set $K=\prod_{i \in I} K_{i}$ containing $S$ such that for each $i \in I$ :

(i) for each $x \in\left[K \cap \triangle_{i}\right] / \prod_{i \in I} D_{i}, A_{i}(x) \cap P_{i}(x) \cap K_{i} \neq \emptyset$;

(ii) for $x \in\left[K / \prod_{i \in I} D_{i}\right], A_{i}(x) \cap K_{i} \neq \emptyset$.

Then an equilibrium point for the game exists.

Proof. For each $i \in I$, define $\phi_{i}: X \rightarrow 2^{X_{i}}$ by

$$
\phi_{i}(x)= \begin{cases}P_{i}(x) \cap A_{i}(x), & \text { if } x \in \triangle_{i} \\ A_{i}(x), & \text { otherwise }\end{cases}
$$

Fix $i$ and $x \in \triangle_{i}$ where $\left(P_{i} \cap A_{i}\right)(x) \neq \emptyset$. There exists an open neighborhood $N_{x}$ of $x$ and a correspondence $\psi_{x}: X \rightarrow 2^{X_{i}}$ such that (1) for each $x^{\prime} \in N_{x}, x_{i}^{\prime} \notin$ $\operatorname{co}_{x}\left(x^{\prime}\right)$ and $P_{i}\left(x^{\prime}\right) \cap A_{i}\left(x^{\prime}\right) \subset \psi_{x}\left(x^{\prime}\right)$, (2) for each $y \in X_{i}, \psi_{x}^{-1}(y)$ is compactly open. Since $A_{i}$ has locally compactly open lower section at $x$, there is an open set $W_{x}$ containing $x$ such that $A_{i}^{-1}(y) \cap W_{x}$ is compactly open for each $y \in X_{i}$. Let $O_{x}=N_{x} \cap W_{x}$. Define $\varphi_{x}: X \rightarrow 2^{X_{i}}$ by

$$
\varphi_{x}\left(x^{\prime}\right)= \begin{cases}\psi_{x}\left(x^{\prime}\right) \cap A_{i}\left(x^{\prime}\right), & \text { if } x^{\prime} \in \triangle_{i} \cap O_{x} ; \\ A_{i}\left(x^{\prime}\right), & \text { if } x^{\prime} \in O_{x} / \triangle_{i} ; \\ \emptyset, & \text { if } x^{\prime} \in X / O_{x} .\end{cases}
$$

Then $\varphi_{x}$ is of class $L_{S}$ and is an $L_{S}$-majorant of $\phi_{i}$ at $x$. For each $x \in X / \triangle_{i}$, since $A_{i}$ is $L_{S}$-majorized at $x$ and $\triangle_{i}$ is closed, it follows that $\phi_{i}$ is $L_{S}$-majorized at $x$. Thus $\phi_{i}$ is $L_{S}$-majorized everywhere.

For each $i \in I$, define $\phi_{i}^{\prime}: X \rightarrow 2^{X}$ by $\phi_{i}^{\prime}(x)=\left\{x^{\prime} \in X \mid x_{i}^{\prime} \in \phi_{i}(x)\right\}$. Since $\phi_{i}$ is $L_{S}$-majorized everywhere, $\phi_{i}^{\prime}$ is $L_{S}$-majorized everywhere for each $i \in I$.

Define $\Lambda: X \rightarrow 2^{I}$ by $\Lambda(x)=\left\{i \in I \mid \phi_{i}^{\prime}(x) \neq \emptyset\right\}$. Define $U: X \rightarrow 2^{X}$ by $U(x)=\bigcap_{i \in \Lambda(x)} \phi_{i}^{\prime}(x)$ for each $x \in X$. Suppose $U(x) \neq \emptyset$. From assumption (6), choose $i \in \Lambda(x)$ with $x \in$ int $_{X}\left\{x \in X \mid\left(P_{i} \cap A_{i}\right)(x) \neq \emptyset\right\}$. Then $\phi_{i}^{\prime}(x) \neq \emptyset$ and there exists an open neighborhood $G_{x}$ of $x$ and $T_{x}: X \rightarrow 2^{X}$ such that (1) for each $x^{\prime} \in G_{x}, \phi_{i}^{\prime}\left(x^{\prime}\right) \subset T_{x}\left(x^{\prime}\right), x^{\prime} \notin \operatorname{co} T_{x}\left(x^{\prime}\right)$ and (2) for each $y \in X, T_{x}^{-1}(y)$ is compactly open. Thus there exists an open subset $O \subset G_{x}$ containing $x$ such that $\phi_{i}^{\prime}\left(x^{\prime}\right) \neq \emptyset$ for each $x^{\prime} \in O$. Then $U$ is majorized by $T_{x}$ on $O$. This implies that $U$ is $L_{S}$-majorized in $X$.

Moreover, for each finite set $S \subset X$ there exists a compact convex set $K=$ $\prod_{i \in I} K_{i}$ containing $S$ such that for each $i \in I$ : If $x \in\left[K \cap \triangle_{i}\right] / \prod_{i \in I} D_{i}$, then $A_{i}(x) \cap P_{i}(x) \cap K_{i} \neq \emptyset$; if $x \in\left[K / \prod_{i \in I} D_{i}\right]$, then $A_{i}(x) \cap K_{i} \neq \emptyset$. From above, we have $\phi_{i}(x) \cap K_{i} \neq \emptyset$ for each $x \in\left[K / \prod_{i \in I} D_{i}\right]$ and each $i \in I$. 
Thus $U(x) \cap \prod_{i \in I} K_{i} \neq \emptyset$ for each $x \in\left[K / \prod_{i \in I} D_{i}\right]$. By Theorem 2 , there exists $\tilde{x} \in \prod_{i \in I} D_{i}$ such that $U(\tilde{x})=\emptyset$. Then $\phi_{i}(\tilde{x})=\emptyset$ for each $i \in I$. From assumption (2), $\tilde{x} \in \bigcap_{i \in I} \triangle_{i}$. Hence $\tilde{x}_{i} \in B_{i}(\tilde{x})$, and $P_{i}(\tilde{x}) \cap A_{i}(\tilde{x})=\emptyset$ for each $i \in I$.

Corollary 2. Let $X=\prod_{i \in I} X_{i}$ and $\left(X_{i}, A_{i}, B_{i}, P_{i}\right)_{i \in I}$ be an abstract economy. Suppose the following conditions are satisfied:

(1) $X_{i}$ is a nonempty convex subset of a Hausdorff topological vector space $E_{i}$ for each $i \in I$;

(2) for each $x \in X, A_{i}(x)$ is nonempty and $\operatorname{co} A_{i}(x) \subset B_{i}(x)$ for each $i \in I$;

(3) $\triangle_{i}:=\left\{x \in X \mid x_{i} \in B_{i}(x)\right\}$ is closed in $X$ for each $i \in I$;

(4) $A_{i}: X \rightarrow 2^{X_{i}}$ has compactly open lower sections for each $i \in I$;

(5) $A_{i} \cap P_{i}: X \rightarrow 2^{X_{i}}$ is $L_{S}$-majorized in $\triangle_{i}$ for each $i \in I$;

(6) $\bigcap_{i \in I}\left\{x \in \triangle_{i} \mid\left(P_{i} \cap A_{i}\right)(x)=\emptyset\right\}=\bigcap_{i \in I} c l\left\{x \in \triangle_{i} \mid\left(P_{i} \cap A_{i}\right)(x)=\emptyset\right\}$;

(7) there exist a nonempty compact convex subset $K_{0}$ of $X$ and a nonempty compact subset $D$ of $X$ such that for each $y \in X / D$ there is an $x \in$ $\operatorname{co}\left(K_{0} \cup\{y\}\right)$ with $x_{i} \in \operatorname{co}\left(A_{i}(y) \cap P_{i}(y)\right)$ for all $i \in I$.

Then an equilibrium point for the game exists.

Remark 2. Assumptions (3), (5) and (6) in Corollary 2 improve the relative assumptions in Theorem 4 of Ding and Tan [7], Theorem 3.5 of Ding and Yuan [8], and Theorem 2.3 of Yuan and Tarafdar [16]. Also assumption (7) in Corollary 2 generalizes these theorems without paracompactness or one-point intersection.

In Corollary 2, let $A_{i}(x)=B_{i}(x)=X_{i}$ for each $x \in X$. Then we have the following:

Corollary 3. Let $X=\prod_{i \in I} X_{i}$ and $\left(X_{i}, P_{i}\right)_{i \in I}$ be a qualitative game. Suppose the following conditions are satisfied:

(1) $X_{i}$ is a nonempty convex subset of a Hausdorff topological vector space $E_{i}$ for each $i \in I$;

(2) $P_{i}: X \rightarrow 2^{X_{i}}$ is $L_{S}$-majorized for each $i \in I$;

(3) $\bigcap_{i \in I}\left\{x \in X \mid P_{i}(x)=\emptyset\right\}=\bigcap_{i \in I} \operatorname{cl}\left\{x \in X \mid P_{i}(x)=\emptyset\right\}$;

(4) there exist a nonempty compact convex subset $K_{0}$ of $X$ and a nonempty compact subset $D$ of $X$ such that for each $y \in X / D$ there is an $x \in$ $\operatorname{co}\left(K_{0} \cup\{y\}\right)$ with $x_{i} \in \operatorname{co}\left(P_{i}(y)\right)$ for all $i \in I$.

Then an equilibrium point for the game exists.

Let $\Gamma=\left\{N,\left(X_{i}\right)_{i \in N},\left(p_{i}\right)_{i \in N}\right\}$ be an $n$-person game in normal form, where $N=\{1,2, \cdots, n\}$ is the set of agents, $X_{i}$ is a nonempty compact convex subset of a Hausdorff topological vector space, $X_{i}$ is the set of strategies of the $i$ th agent, $p_{i}$ is a function from the product $X=\prod_{i \in N} X_{i}$ into the real numbers $R$, and $p_{i}$ represents the utility (payoff or cost) function of $i$ th agent. For each $i$, let $X_{-i}=\prod_{j \neq i} X_{j}$. For $x \in X$ and $i \in N, x=\left(x_{i}, x_{-i}\right)$. We say that a vector $x$ is a pure-strategy Nash equilibrium if, for each $i \in N, x_{i} \in X_{i}$ and for each $t \in X_{i}$,

$$
p_{i}\left(x_{i}, x_{-i}\right) \leq p_{i}\left(t, x_{-i}\right) .
$$

For each $i$, define a correspondence $P_{i}: X \rightarrow 2^{X_{i}}$ by $P_{i}(x)=\left\{y_{i} \in X_{i}\right.$ | $\left.p_{i}\left(y_{i}, x_{-i}\right)<p_{i}(x)\right\}$. If $p_{i}\left(., x_{-i}\right)$ is quasiconvex on $X_{i}$, then $P_{i}(x)$ is convex and $x_{i} \notin P_{i}(x)$ for all $x \in X$. If $p_{i}$ is continuous, then $P_{i}$ has an open graph. Thus we can replace preference correspondences $\left\{P_{i}\right\}_{i \in N}$ in Corollary 3 by cost functions $\left\{p_{i}\right\}_{i \in N}$ and have the following: 
Corollary 4. Let $X=\prod_{i \in N} X_{i}$ and $\left\{N,\left(X_{i}\right)_{i \in N},\left(p_{i}\right)_{i \in N}\right\}$ be an n-person game. Suppose the following conditions are satisfied:

(1) $X_{i}$ is a nonempty convex subset of a Hausdorff topological vector space $E_{i}$ for each $i \in N$;

(2) the cost function $p_{i}: X \rightarrow R$ is continuous in $X$ and the function $p_{i}\left(., x_{-i}\right)$ is quasiconvex on $X_{i}$ for each $x$ and each $i \in N$;

(3) there exist a nonempty compact convex subset $K_{0}$ of $X$ and a nonempty compact subset $D$ of $X$ such that for each $y \in X / D$ there is an $x \in$ $c o\left(K_{0} \cup\{y\}\right)$

$$
\sup _{i \in N}\left(p_{i}\left(x_{i}, y_{-i}\right)-p_{i}(y)\right)<0 .
$$

Then the game admits a pure-strategy Nash equilibrium.

\section{REFERENCES}

[1] K. J. Arrow and G. Debreu, Existence of an equilibrium for a competitive economy, Econometrica 22 (1954), 265-290. MR 17:985e

[2] A. Borglin and H. Keiding, Existence of equilibrium actions and of equilibrium: A note on the "new" existence theorems, J. Math. Econom. 3 (1976), 313-316. MR 56:2451

[3] S. Y. Chang, A generalization of KKM principle and its applications, Soochow J. Math. 15 (1989), no. 1, 7-17. MR 91a:54025

[4] S. Y. Chang, On the Nash equilibrium, Soochow J. Math. 16 (1990), no. 2, 241-248. MR 91m:90028

[5] G. Debreu, A social equilibrium existence theorem, Proc. Nat. Acad. Sci. USA 38 (1952), 886-893. MR 14:301c

[6] X. P. Ding, W. K. Kim, and K. K. Tan, Equilibria of noncompact generalized games with $L^{*}$-majorized preference correspondences, J. Math. Anal. Appl. 164 (1992), 508-517. MR 93d:90014

[7] X. P. Ding and K. K. Tan, On equilibria of noncompact generalized games, J. Math. Anal. Appl. 177 (1993), 226-238. MR 94d:90075

[8] X. P. Ding and X. Z. Yuan, The study of existence of equilibria for generalized games without lower semicontinuity in locally topological vector spaces, J. Math. Anal. Appl. 227 (1998), 420-438. MR 99k:90066

[9] D. Gale and A. Mas-Colell, On the role of complete, transitive preferences in equilibrium theory, in: G. Schwödiauer, ed., Equilibrium and disequilibrium in economic theory (Reidel, Dordrecht) (1978), 7-14.

[10] W. K. Kim, Existence of maximal element and equilibrium for a nonparacompact $N$-person game, Proc. Amer. Math. Soc. 116 (1992), no.3, 797-807. MR 93a:90083

[11] J. F. Nash, Equilibrium points in n-person games, Proc. Nat. Acad. Sci. USA 36 (1950), 48-49. MR 11:192c

[12] W. Shafer and H. Sonnenschein, Equilibrium in abstract economies without ordered preferences. J. Math. Econom. 2 (1975), no.3, 345-348. MR 53:2315

[13] K. K. Tan and X. Z. Yuan, Existence of equilibrium for abstract economies. J. Math. Econom. 23 (1994), no.3, 243-251. MR 95h:90024

[14] N. C. Yannelis and N. D. Prabhakar, Existence of maximal elements and equilibria in linear topological spaces, J. Math. Econom. 12 (1983), 233-245. MR 87h:90061a

[15] N. C. Yannelis, Maximal elements over noncompact subsets of linear topological spaces, Economics Letters 17 (1985), 133-136. MR 86i:90011

[16] X. Z. Yuan and E. Tarafdar, Existence of equilibria of generalized games without compactness and paracompactness, Nonlinear Anal. 26 (1996), no. 5, 893-902. MR 96k:90019

Department of Mathematics, Soochow University, Taipei, Taiwan, Republic of China E-mail address: sychang@math.scu.edu.tw 